\title{
Adriana Chammas'
}

\section{O perfil do consumidor criativo}

\author{
The creative consumer profile
}

\begin{abstract}
Resumo
A economia criativa desponta como a terceira maior indústria no cenário mundial. Relacionada à inovação, é fundamentada no poder intelectual, no desenvolvimento sustentável e na propulsão de talentos individuais. Com os avanços da tecnologia, a comunicação entre os consumidores (usuários) transformou o mercados e comportamentos. O consumo colaborativo evidencia essa alteração ao apontar para as modificações nesse cenário, onde consumidores (usuários) utilizam a tecnologia associada à criatividade para fazer amizades, gerar renda, criar empregos e economizar. O objetivo desse artigo é identificar o perfil do consumidor (usuário) na economia criativa atual, influenciado pelos canais de comunicação digitais e pelos avanços do consumo colaborativo. Além disso, pretende-se mapear algumas das motivações diretas dos consumidores na economia criativa atual e identificar tendências de negócios que se dediquem a explorá-las.
\end{abstract}

Palavras-chave: Economia criativa. Consumo colaborativo. Usuário.

\begin{abstract}
The creative economy has emerged as the third largest industry in the world scenario. Related to innovation, it is based on the intellectual power, sustainable development and individual talents. With advances in technology, communication between consumers (users) changed markets and behaviors. The collaborative consumption shows that by pointing out changes in this scenario, where consumers (users) use the technology associated with creativity to make personal and professional relations, generate income, create jobs and save money. The aim of this paper is identify the user profile in the current creative economy, driven by digital communication channels and the progress of the collaborative consumption.
\end{abstract}

Keywords: Creative Economy. Collaborative Consumption. Users.

\footnotetext{
${ }^{1}$ Empreteca desde 2004, pelo Sebrae, Coppe (UFRJ) e Designer pela ESPM Rio. Graduada em Design com ênfase em Marketing. Mestre em Design pela Pontifícia Universidade Católica, a PUC-Rio na linha de pesquisa Ergonomia e Usabilidade de Sistemas Informacionais e Doutoranda na mesma linha de pesquisa. Consultoria em Usabilidade em diversas empresas especializadas em pesquisas no Rio, em São Paulo e em Curitiba. Professora no curso de especialização em Ergonomia, no CCE da PUC-Rio. Integrante do LEUI, o Laboratório de Ergodesign e Usabilidade de Interfaces da PUC-Rio. UX Expert no programa do Google Developers Experts desde 2014.
} 


\section{Introdução}

A economia clássica é arraigada em estruturas sólidas e, apesar de poderosa e lucrativa, arca com custos e entraves palpáveis. Tradicionalmente orientadas por alicerces como os 4Ps - produto, preço, ponto e promoção - as decisões do mercado atual têm mudado significativamente sua fundamentação. A internet democratizou barreiras antes intransponíveis de distribuição e acesso a conteúdo, exterminando canais de mídia tradicionais. A forma de alcançar os consumidores (usuários) há muito não é a mesma e os canais de comunicação e distribuição tão pouco. Com mais de 2 bilhões de usuários de smartphones, a informação é trocada de forma exponencial e muitos setores tentam entender as mudanças desse cenário para acompanhá-las. As decisões são baseadas em forte investimento em capital intelectual e cada vez mais a competência central é a criatividade. Relacionada à inovação, a economia criativa assume papel decisivo no cenário mundial. A economia criativa está posicionada como a terceira maior indústria mundial, atrás da indústria do petróleo e da indústria de armamentos, nessa ordem (SEBRAE, 2012).

Esse artigo tem por objetivo identificar o perfil do consumidor (usuário) na economia criativa, influenciado pelos canais de comunicação digitais e revelado pelos evidentes avanços do consumo colaborativo.

\section{Criatividade na Economia}

A Unesco (2013) define economia criativa como

atividades relacionadas à criação, produção e comercialização de conteúdos intangíveis e culturais em sua natureza, protegidos pelos direitos autorais e que podem tomar a forma de bens e serviços. São intensivos em trabalho e conhecimento e estimulam a criatividade e incentivam a inovação dos processos de produção e comercialização.

Já o British Council (2006), unidade britânica internacional dedicada a relações culturais e oportunidades educacionais, define a economia criativa como "a criação de divisas e de empregos por meio da geração e exploração da atividade intelectual". Essa definição abrange a transmissão, a difusão, a distribuição e o licenciamento das artes, propaganda, arquitetura, artesanato, design, produção visual física ou digital, softwares, jogos de computador, música, artes cênicas, publicações, televisão, rádio e outras mídias.

Entende-se a economia criativa como a soma de talento, habilidade, criatividade e oportunidade que, empregada de maneira estratégica, resulta na exploração da propriedade intelectual gerando renda e empregos. Também associada ao termo "economia da cultura", a economia criativa é propagadora do desenvolvimento sustentável e da proliferação de talentos individuais. Por consistir em uma atividade econômica diretamente relacionada ao mundo das artes, de expoentes da mídia digital, softwares de entretenimento interativo e outras mídias - notadamente transmissão de imagem, filme e televisão, música etc., a categoria abrange atividades diretamente relacionadas à Arquitetura e ao Design. Entre essas atividades elen- 
cam-se a comunicação visual, design de produto, design de interiores, design de moda, ou seja, produtos e serviços baseados no conhecimento e na atividade intelectual. Em especial o Design, pela multiplicidade disciplinar característica, permeia os conceitos básicos apresentados anteriormente e se faz presente em múltiplas facetas desse novo cenário, onde despontam produtos e produções que ultrapassam limites clássicos com os avanços da tecnologia.

A fronteira entre as empresas, antes fortemente demarcadas, perdeu espaço para a associação entre produtos e serviços oferecidos em nome da experiência do usuário - e do consequente fortalecimento das marcas. Exemplos de parcerias que mesclam empresas são amiúde: a Salinas ${ }^{\circledR}$, loja de moda praia carioca que fez parceria com a Ipanema, que fabrica e distribui chinelos com as estampas exclusivas da Salinas. As sandálias Ipanema ${ }^{\circledR}$ também fabricam e distribuem chinelos de dedos para a Lenny, outra emblemática marca de moda praia. Vale lembrar que as sandálias Ipanema ${ }^{\circledR}$ têm a segunda colocação em vendas de chinelos de dedo do país, atrás das Havaianas, líder de mercado. O Google formou uma parceria com a banda de rock Arcade Fire para promover novas funcionalidades em um de seus produtos. O projeto consiste em permitir que o consumidor/usuário adicione efeitos visuais no filme "Just a Reflektor" durante a execução da música da banda. O consumidor/ usuário segura seu smartphone ou tablet em frente à câmera de seu computador e consegue interagir com o filme sem usar nem o mouse, nem o teclado.

O Uber faz associações esporádicas com empresas como o TTBurguer $®$, restaurante especializado no prato que o batiza. O Uber entregou no dia 23 de novembro de 2015 uma refeição gratuita na casa dos usuários que entrassem em contato com a empresa pelo aplicativo. No dia 20 de fevereiro de 2016, o Uber ofereceu para os consumidores/usuários do Uber/Summer uma bolsa com produtos essenciais para a praia e, entre os produtos, garrafinhas de água Evian. O próprio Uber é uma releitura do serviço de transportes. Há muito acomodado, o táxi convencional tem precisado adaptar seus serviços desde o surgimento do concorrente. Frotas carentes de conforto, segurança e de atendimento de ponta perderam espaço para os anseios do novo consumidor, que espera ser (bem) servido, compartilha boas experiências e promove marcas com a força do boca a boca. Pergunta-se então o que a não delimitação de fronteiras pode significar para o mercado? Como é esse novo perfil de consumidor/usuário que se revela?

Diferente da economia clássica, a economia criativa possibilita que oportunidades antes inimagináveis se tornem realidade a custos ínfimos e com extrema celeridade. Existem evidências de startups expoentes que ultrapassaram as barreiras do Vale do Silício, considerado berço da inovação. Podem ser conferidos exemplos como a Apple, o Google, a Microsoft entre muitas outras, todas empresas diminutas que cresceram rapidamente e se subdividiram para multiplicar a esfera criativa. As startups seguem o padrão colaborativo e são em geral promovidas por programas de aceleração e investidores. Custeados pelos aceleradores de negócios, talentos se reúnem interessados em encontrar soluções para seus consumidores (usuários) através da troca de conhecimento. O design, disciplina que permeia muitas outras, transborda a máxima "extrair o máximo do mínimo" quando esclarece que, na prática, a criatividade, as habilidades e os talentos pessoais são maximizados quando equipes multidisciplinares proporcionam a mágica do conjunto. Essas equipes ge- 
ram valor real, seja em forma de oportunidades de trabalho, geração de renda e efetivação da propriedade intelectual. Mas onde o Design realmente faz diferença é na criação e implantação de novos modelos de negócio, de novas possibilidade de operação, de novas formas de quebrar paradigmas.

O mercado há muito percebeu que os consumidores têm mudado seu comportamento, seja consequência das crises mundiais, seja pelos efeitos ecológicos que o planeta tem apresentado. Uma das muitas evidências da alteração de comportamento do consumidor foi o surgimento do consumo colaborativo, baseado no aproveitamento inteligente dos bens e serviços. O consumo colaborativo faz uso da criatividade para fazer amizades, gerar renda, criar empregos e ainda economizar. Através das redes sociais, consumidores compartilham produtos e serviços, trocam ativos tangíveis ou não, compartilham espaços, tarefas, contatos etc. As redes formadas na internet possibilitam ainda a troca, a revenda, a locação, empréstimos e doação dos produtos de produtos ou serviços. Além desses benefícios, os recursos naturais são preservados com a diminuição do material de descarte (BOTSMAN; ROGERS, 2012).

A força que o consumo colaborativo ganhou nos últimos vinte anos aponta para a saturação do consumidor em relação ao excesso de consumo de poucos anos atrás. Efeitos positivos, como o prazer da aquisição ou o de ser servido, vêm sendo substituídos pelo prazer de pagar apenas pelo que é consumido - e enquanto é consumido. Consumidores ponderam hoje quais efeitos negativos o consumo pode trazer ao planeta, que encargos estarão vinculados à aquisição do bem, quanta energia e tempo serão gastos nesse processo e quanto espaço físico será necessário para armazená-lo (WORLDWIDE, 2014). Esse movimento delineado pelo consumo colaborativo aponta para a necessidade de adequar novos negócios ao modelo mental dos consumidores. Quem são eles? Como pensam? Que objetivos eles têm? Por quê? São algumas das muitas perguntas que possibilitarão a releitura do novo comportamento do consumidor. É exatamente nesse ponto que o Design encontra espaço para repensar toda a cadeia produtiva. $\mathrm{O}$ bom design deve auxiliar as empresas a realizarem suas estratégias a contento. A oportunidade latente é a de repensar modelos de negócio, muito além de produtos novos.

Interessados no tema, Botsman e Rogers (2012) classificaram as diversas formas de consumo colaborativo em três tipos: sistemas de serviços de produtos, mercados de redistribuição e estilo de vida colaborativo:

Como sistemas de serviços de produtos, entende-se utilizar produtos sem possuir os mesmos (aluguel), sendo esses produtos de empresas ou de pessoas físicas. Alguns exemplos desse tipo de sistema as bicicletas locadas por um curto espaço de tempo, como é o caso das bicicletas do Banco Itaú, disponíveis em diversos pontos da cidade. Botsman e Rogers (2012) apontam que nos Estados Unidos de 1940 seria impensável compartilhar um carro com outras pessoas. Além da questão do valor imobilizado e de todas as restrições a respeito da segurança, havia ainda a dificuldade de encontrar os contatos certos para viabilizar o negócio. Os autores salientam que com o advento da internet e das redes sociais esses obstáculos foram superados e a coordenação de atividades entre usuários se tornou fácil. Esse avanço tornou possível não só o compartilhamento de carros, mas de praticamente qualquer produto, seja ele físico ou não. Hoje é possível compartilhar ativos intangíveis como 
tempo, tarefas, experiências etc.

O RelayRides repensa o modelo de negócios das locadoras de veículos e possibilita que proprietários de automóveis aluguem seus veículos enquanto não estão usando-os. É o redesign do um modelo de transporte. A ideia da empresa transforma um ônus - o pagamento de horas de estacionamento, por exemplo, em um bônus - o valor das horas de locação do automóvel. A plataforma de compartilhamento de veículos RelayRides angariou o valor de U\$35 milhões em 2015 para aprimorar seu negócio (STRAUSS, 2014). A RelayRides capta $25 \%$ de cada transação enquanto o proprietário do veículo faz em média U\$200 por mês de renda extra (STRAUSS, 2014). Sites como o Zilok ou o Rentoid possibilita as pessoas alugarem, além de veículos, qualquer objeto que tenham em casa, como vitrolas, casas, tratores etc. Esse tipo de sistema de serviços de produtos gera benefício para os envolvidos no negócio. Gera renda para os locadores enquanto favorece a economia dos locatários, já que não precisarão pagar pelos encargos da propriedade, manutenção, consertos, multas e seguro. Além disso, o sistema apresenta vantagens ambientais ao maximizar a utilidade dos produtos.

Os mercados de redistribuição são redes sociais que permitem que mercadorias usadas sejam redistribuídas. As mercadorias são trocadas diretamente, até sem a necessidade de transação monetária, alguns casos. Também podem ser vendidas ou trocadas por pontos, ou mesmo uma mistura desses tipos de transação. As transações são feitas online e costumam ser feitas por pessoas que não se conhecem pessoalmente. Os mercados de redistribuição estimulam a revenda e reutilização itens sem uso, em vez de encaminhá-los para o descarte. Esse novo modelo de negócios contesta a tradicional relação entre produtor, varejista e consumidor.

Exemplos desse tipo de mercado são: os sites de vendas de usados e-Bay, Flippid, Mercado Livre e OLX; sites de trocas livres como o Freecycle, Kashless e Around Again; sites de trocas de produtos por produtos semelhantes a exemplo do Thred-Up, MakeupAlley, Swapstyle, Toyswap e 'N' Dig Swap; sites de trocas por pontos Barterquest e UISwap; e ainda sites que misturam tipos de troca, como o SCoodle e Craigslist.

Estilo de vida colaborativo são os sistemas de troca de ativos intangíveis entre pessoas com interesses comuns. Nesses sistemas são negociados tempo, espaço, habilidades e contatos, por exemplo. Em muitos casos o grau de confiança entre as partes é decisivo, pois a interação é feita entre pessoas e a confiança deve ser mútua. Sistemas que viabilizam o estilo de vida de colaborativo podem ser sites de compartilhamento de espaços de trabalho, como o Citizen Space e Hub Culture; o Neighborrow, um site de compartilhamento de bens entre vizinhos; sites de compartilhamento de tarefas, tempo, serviços e recados como o DaveZillion; o site de compartilhamento de jardins Urban Garden Share; o site de compartilhamento de vagas de estacionamento Just Park; sites de compartilhamento de empréstimos sociais Zopa e Prosper; entre diversos outros.

Ao mesclar as formas de consumo colaborativo categorizadas por Botsman e Rogers (2012), startups como Airbnb, Stayzilla, Couchsurfing entre outras, fazem a releitura do padrão de hotelaria tradicional. As startups possibilitam que qualquer proprietário de imóvel possa lucrar com uma área mal aproveitada dentro de casa. 
O redesign da hotelaria estendeu possibilidades e transformou conceitos na mente do consumidor. As viagens se transformaram em aventuras: turistas que antes se preocupavam muito mais com o conforto dos hotéis, hoje se dedicam a explorar a experiência das cidades que visitam, a vivenciar a cultura local - e ainda fazer amizades que podem durar uma vida!

O Homeshare International ${ }^{(2015)}$ possibilita que pessoas que não se conheçam compartilhem a convivência em troca de benefícios mútuos. Proprietários atarefados ou senhores de idade trocam o valor que seria pago pela hospedagem por serviços corriqueiros, como levar os cachorros para passear ou ir ao mercado, por exemplo. Os hóspedes fornecem suporte e companhia em troca de acomodação, o que vai além do conceito de hotelaria. Além de completarem seus afazeres, os proprietários renovam as energias da casa recebendo hóspedes de diferentes culturas e nacionalidades. De acordo com os comentários dos consumidores (usuários) do produto, o Homeshare ${ }^{\circledR}$ acaba por promover relações entre gerações e realçar que todos têm necessidades e algo para oferecer.

Ainda na linha da troca, o Home Exchange ${ }^{\circledR}$ (2016) sugere que os grupos troquem de casa pelo mesmo período, em vez de receber por isso. Os consumidores (usuários) podem planejar sua viagem e, além da experiência de conhecer um local, terão a experiência de se sentirem moradores e entenderem a cultura local sob outra ótica. Ambos os grupos saem ganhando, tanto em termos financeiros quanto em termos afetivos. A avaliação dos hóspedes ou o compartilhamento da informação sobre o imóvel ou sobre os proprietários credencia e sustenta o serviço.

Para Botsman e Rogers (2012) as formas de consumo colaborativo explanadas acima apontam para as várias motivações diretas dos consumidores (usuários): economizar tempo, economizar espaço, economizar de dinheiro, ganhar dinheiro e ainda, mas não menos importante, aumentar a rede social e profissional.

Glind (2013) classificou o consumo colaborativo como um novo movimento socioeconômico e determinou que os principais fatores que contribuem para a adoção do consumo colaborativo corroboram com os fatores encontrados pelos pesquisadores Botsman e Rogers (2012). São eles: as facilidades que a tecnologia proporciona, a valorização da vida em comunidade, as preocupações a respeito do equilíbrio ambiental e o consumo consciente. Glind (2013) concluiu que quanto maior a idade, menor a propensão a aceitar o consumo colaborativo. Sua pesquisa aponta que apesar de existir uma relação entre disposição para o consumo colaborativo e a idade, o movimento não está restrito aos mais jovens, nem se relaciona à experiência com produtos tecnológicos. A pesquisa também evidencia o poder que a tecnologia ofereceu ao consumidor / usuário. Labrecque et al (2013) apresentam a evolução do poder dos consumidores com os ambientes online. Os autores dividem categorizam em quatro as fontes de poder dos consumidores online: (1) demanda, (2) informação, (3) network ${ }^{2}$ e (4) crowd $^{3}$. As duas primeiras fontes de poder são relacionadas

\footnotetext{
2 Network é uma rede de telecomunicação que permite que dois ou mais computadores troquem dados. As conexões são estabelecidas através de cabos ou redes sem fio. Utilizado em sentido figurado pelo autor, o termo significa uma rede de relacionamentos entre pessoas com propósitos sociais ou profissionais.

${ }^{3}$ A expressão americana "crowd" significa um número expressivo de pessoas reunidas, em geral de forma desorganizada e desregrada.
} 
ao indivíduo, ao passo que as duas últimas, às redes sociais.

(1) O poder da demanda: a internet possibilitou a comparação de produtos e preços entre produtos e o acesso a uma maior variedade de ofertas. O surgimento dos sistemas de busca, a familiarização com as interfaces gráficas e o acesso ao mercado alterou a demanda das empresas.

(2) O poder da informação é bipartido entre a capacidade do consumidor (usuário) consumir conteúdo e a capacidade de gerar conteúdo. O consumo de conteúdo é diretamente relacionado à facilidade que o consumidor tem em conseguir informações sobre produtos e serviços. A capacidade de gerar conteúdo possibilita que o consumidor (usuário) aumente o potencial de suas opiniões individuais e a de seus pares, e por conseguinte amplie seu alcance individual para influenciar o mercado. O poder gerado pelo cruzamento de informações pode significar o sucesso ou o fracasso de uma marca, mesmo já consagrada.

(3) Network: surgimento das redes sociais possibilitou aos consumidores / usuários interagirem com o conteúdo publicado na internet. Esse poder pode ser aplicado para construir reputações, alterar comportamentos e influenciar mercados através da edição e distribuição desse conteúdo. Essa fonte de poder está relacionada à possibilidade de outras pessoas adicionarem valor ao conteúdo original.

(4) Crowd: Com os avanços na tecnologia e da infraestrutura de dados, o acesso, a criação e a disseminação de conteúdos online estão cada vez mais rápidos e fáceis. A fonte de poder do consumidor / usuário baseada em crowd fundamenta-se nos grupos e comunidades online, onde pessoas com interesses similares fomentam formas de compartilhamento entre pessoas e grupos. O crowd, poder baseado na mobilização de muitas pessoas, reune, mobiliza e estrutura recursos que beneficiem os indivíduos e os grupos.

\section{Conclusão}

$\mathbf{E}$

ntende-se a partir do exposto que economia criativa pode ser potencializada através o redesign dos negócios, mas que somente com uma precisa compreensão das necessidades e desejos do consumidor (usuário) poderão ser estabelecidos os requisitos do produto / serviço. Dorsey (2011) enfatiza que para encontrar a solução correta para o produto / serviço, é fundamental entender a fundo os desejos, emoções do consumidor (usuário) e o contexto onde o produto / serviço será utilizado. Entende-se que conhecer a fundo o consumidor (usuário) é ponto premissa para que as empresas atinjam seus objetivos estratégicos. Nesse artigo foram mapeadas algumas das motivações diretas dos consumidores (usuários): economia de tempo, espaço e dinheiro, lucratividade e aumento da rede social e profissional. Nessa era, a da criatividade, o consumidor (usuário) tem acesso a informação, consegue se comunicar, fortalecer relações e ainda tem o poder de movimentar e mobilizar grupos e comunidades.

O avanço do consumo colaborativo ilustra que a tendência das pessoas é utilizar produtos e serviços de forma inteligente e rentável, sem adquirí-los. A revenda, locação, empréstimos, doação e/ou reutilização de itens sem uso ou parcamente 
aproveitados são uma pequena amostra do poder que as comunidades formadas na internet dão ao consumidor (usuário). O relatório Worldwide (2014) corrobora com a tendência ao identificar o surgimento de uma consciência de consumo que busca escolhas mais alinhadas com a valores pessoais, em busca de substituir a culpa pelo consumo exagerado. 0 "novo" perfil consome o que realmente é necessário, se preocupa com a reciclagem e reaproveitamento, com os danos ao planeta (por conseguinte ao coletivo), se preocupa mais com a qualidade dos produtos e menos com a quantidade, prefere consumir produtos produzidos na localidade (para incentivar efeitos positivos na comunidade), e opta por comprar produtos com preços mais baixos. Os termos colaborar e compartilhar são recorrentes e colocam a vida em comunidade em primeiro plano. As mudanças de comportamento dos consumidores (usuários) podem significar desafios, mas certamente podem ser lidas como oportunidades. 


\section{Referências}

AIRBNB. São Francisco, 2008. Disponível em: <https://www.airbnb.com>. Acesso em: 20 fev. 2016.

GOOGLE CHROME EXPERIMENTS / ARCADE FIRE. Disponível em: <https://www.chromeexperiments. com/arcadefire/> e <http://mashable.com/2010/08/30/arcade-fires-chrome-video/\#QFsuuEv90ZqO> Acesso em: 20 fev. 2016.

BOTSMAN, Rachel; ROGERS, Roo. What is mine is yours: the rise of collaborative consumption. New York: Harper Collins, 2010.

BRITISH COUNCIL. British Council Anual Report. London, 2005. Disponível em: <https://www.britishcouncil.org/sites/default/files/annual_report_2005_2006.pdf> Acesso em: 20 fev. 2016.

BRITISH COUNCIL. Creative economy. London, 2014. Disponível em: <http://creativeconomy.britishcouncil.org/>. Acesso em: 20 fev. 2016.

COUCHSURFING. Los Angeles, 2016. Disponível em <https://www.couchsurfing.com/>. Acesso em: 20 fev. 2016.

CREATIVE industries. In: Wikipedia, 2016. Disponível em: <http://en.wikipedia.org/wiki/Creative_ industries>. Acesso em: 20 fev. 2016.

GLIND, P. V. The consumer potential of collaborative consumption. 2013. Disponível em: <http:// www.collaborativeconsumption.com/2013/08/20/what-is-the-consumer-potential-of-collaborative-consumption-answers-from-amsterdam/>. Acesso em: 20 fev. 2016.

HAVAS WORLDWIDE. Prosumer report: the new consumer and the sharing economy. Nova York: Havas Worldwide, 2014.

HOMEAWAY. 2006. Disponível em: <https://www.homeaway.com/>. Acesso em: 20 fev. 2016.

HOMEEXCHANGE. 2016. Disponível em: <www.homeexchange.com>. Acesso em: 20 fev. 2016.

HOMESHARE INTERNATIONAL. 2015. Disponível em: < http://homeshare.org/ > Acesso em: 20 fev. 2016.

HOUSETRIP. 2009. Disponível em: <https://www.housetrip.com/>. Acesso em: 20 fev. 2016.

IBGE. 2016. Disponível em: <http://www.ibge.gov.br/home/default.php>. Acesso em: 20 fev. 2016.

JUSTPARK. 2016. Disponível em: <https://www.justpark.com/>. Acesso em: 20 fev. 2016.

LABRECQUE, L. I. et al. Consumer power: evolution in the digital age. Journal of Interactive Marketing, Nova York, v. 27, n. 4, p. 257-269, nov. 2013.

MCCRACKEN, G. Culture and consumption: a theoretical account of the structure and movement of the cultural meaning of consumer goods. Journal of Consumer Research, Chicago, v. 13, n. 1, p. 71-84, 1986.

MINISTÉRIO DA CULTURA. Plano da Secretaria da Economia Criativa: políticas, diretrizes e ações 2011 a 2014. 2014. Disponível em: <http://www.cultura.gov.br/documents/10913/636523/PLANO+DA+SECRETARIA+DA+ECONOMIA+CRIATIVA/81dd57b6-e43b-43ec-93cf-2a29be1dd071>. Acesso em: 20 fev. 2016.

NIELSEN COMPANY. Under the influence: consumer trust in advertising. New York, 2013. Disponível em: <http://www.nielsen.com/us/en/insights/news/2013/under-the-influence-consumer-trust-inadvertising.html>. Acesso em: 20 fev. 2016.

NIELSEN COMPANY. Is sharing the new buying?: reputation and trust are emerging as new currencies. New York, 2014. Disponível em: <http://www.nielsen.com/content/dam/nielsenglobal/ apac/docs/reports/2014/Nielsen-Global-Share-Community-Report.pdf>. Acesso em: 20 fev. 2016. 
REIS, Carla Fonseca (Org.). Economia criativa como estratégia de desenvolvimento: uma visão dos países em desenvolvimento. São Paulo: Itaú Cultural, 2008.

RENTOID. Australia, 2016. Disponível: em <http://www.rentoid.com/>. Acesso em: 20 fev. 2016.

ROCHA, Angela da; ROCHA, Everardo. Paradigma interpretativo nos estudos de consumo: retrospectiva, reflexões e uma agenda de pesquisas para o Brasil. Revista de Administração de Empresas, São Paulo, v. 47, n.1, p. 71-80, jan./mar. 2007.

ROGERS, E. M. Diffusion of innovations. Nova York: The Free Press, 2003.

ROOMORAMA. Singapore, 2008. Disponível em: <https://www.roomorama.com/>. Acesso em: 20 fev. 2016.

SALINAS MODA PRAIA. Disponível em: < http://www.salinascompras.com.br/category/816> e <http://www.istoedinheiro.com.br/noticias/negocios/20100312/faminto-por-marcas/24566> Acesso em: 20 fev. 2016.

SANDÁLIAS IPANEMA. Disponível em: <http://www.sandaliasipanema.com.br/> e < http://mundodasmarcas.blogspot.com.br/2010/06/ipanema.html >

SEBRAE. Disponível em: <http://www.sebrae.com.br/Sebrae/Portal\%20Sebrae/UFs/RJ/Menu\%20Institucional/Sebrae_SET_nov12_ec_crtv.pdf> Acesso em: 20 fev. 2016.

STAYZILLA. 2016. Disponível em: <https://stayzilla.com/>. Acesso em: 20 fev. 2016.

STRAUSS, Karsten. RelayRides wants you to rent out your car in 2015. Forbes, Jersey, 2014. Disponível em: <http://www.forbes.com/sites/karstenstrauss/2014/12/17/relayrides-wants-you-to-rent-out-your-car-in-2015/\#72c5a465233b>. Acesso em: 20 fev. 2016.

TTBURGUER. Disponível em: < http://www.ttburger.com.br/ > e <https://www.mundodomarketing. com.br/ultimas-noticias/35009/uber-oferece-tt-burger-gratuito-para-clientes-no-rio-de-janeiro. html>

UNESCO. Understanding creative industries: cultural statistics for public-policy making. 2006. Disponível em: < http://portal.unesco.org/culture/es/files/30297/11942616973cultural_stat_EN.pdf/cultural_stat_EN.pdf >. Acesso em: 20 fev. 2016.

UNESCO. Creative economy report 2008: the challenge of assessing the creative economy: towards informed policy-making. 2008. Disponível em: <http://unctad.org/en/docs/ditc20082cer_en.pdf >. Acesso em: 20 fev. 2016.

UNESCO. Creative economy report 2013: <http://www.unesco.org/culture/pdf/creative-economy-report-2013.pdf>. Acesso em: 20 fev. 2016.

ZYLOK. 2007. Disponível em: <http://us.zilok.com/>. Acesso em: 20 fev. 2016. 